\title{
Efectos de los movimientos cíclicos en la productividad española. Análisis agregado y por sectores
}

\author{
Andrés Maroto-Sánchez* \\ Universidad Autónoma de Madrid \\ Juan R. Cuadrado-Roura** \\ Universidad de Alcalá, Madrid
}

\section{Resumen}

Uno de los temas más debatidos en la economía española es el de su productividad. Mientras en la época expansiva se observó un comportamiento negativo de esta variable, en los últimos años de fuerte crisis económico-financiera su evolución ha sido mucho más positiva, al contrario de lo observado en otros países de nuestro entorno. Por esta razón, el presente trabajo trata de analizar el efecto del ciclo económico sobre nuestra productividad desde los años ochenta hasta la presente crisis, usando datos de la Contabilidad Nacional Trimestral (INE) y The Conference Board (TCB), con especial enfoque en la peculiar estructura sectorial de nuestra economía.

Palabras clave: productividad, ciclo económico, filtro HP, estructura productiva.

Clasificación JEL: C50, E32, F44, L80, O40.

\begin{abstract}
One of the most courageous debates on the Spanish economy is the one related to the productivity. While negative trends were observed during expansions, its evolution has turned out significantly positive during the recent years of strong economic and financial crisis. This behaviour has been additionally opposite to the one displayed by other European countries. For this reason, this paper aims to analyze the effects of the economic cycle on the Spanish labour productivity since 1980 onwards, specially focusing on the characteristic industrial structure of our economy. We use data provided by the Conference Board (TCB) and the Quarterly National Accounts (INE).
\end{abstract}

Keywords: productivity, business cycle, HP filter, productive structure.

JEL classification: C50, E32, F44, L80, O40.

\section{Introducción}

La evolución positiva de la productividad constituye un factor clave para el progreso de cualquier economía y para que el bienestar de sus ciudadanos pueda realmente mejorar. A largo plazo, el crecimiento económico de un país exige, para

* Profesor del Departamento de Análisis Económico: Teoría Económica. Investigador del Instituto de Análisis Económico y Social (IAES), Universidad de Alcalá, Madrid.

** Catedrático de Economía Aplicada. Director-Fundador del Instituto de Análisis Económico y Social (IAES). 
que sea sostenido, que vaya acompañado de continuas mejoras en la productividad. Crecimiento y productividad son, en realidad, dos caras de una misma moneda y cualquier país que aspire a que los ingresos medios por habitante crezcan, debería producir más bienes y servicios con el mismo número de trabajadores -mediante mejoras tecnológicas, organizativas y de equipo capital-, o bien lograr que aumentase el número de personas dispuestas a trabajar y hacer que el sistema productivo proporcionara empleo a los desocupados. Pero, lograr un crecimiento continuado de la productividad no es fácil, entre otras cosas porque depende de un amplio conjunto de factores, entre los cuales destacan algunos cuya influencia se considera decisiva: la acumulación de capital, los avances tecnológicos y las mejoras en términos de eficiencia en el uso y combinación de los factores, que generalmente se identifica con la llamada productividad total de los factores.

Un hecho que se comprueba al contemplar la evolución de la productividad en las economías es que las fluctuaciones cíclicas que estas experimentan pueden y suelen afectar a dicha variable. De hecho, la literatura económica dispone desde hace años de numerosos estudios que confirman que la mejora o el mal comportamiento de esta variable puede ser consecuencia de dichas fluctuaciones cíclicas y no tanto, o en absoluto, de que la economía en cuestión haya incrementado los factores básicos a los que ya hemos hecho referencia (trabajo, capital, tecnología), ni que haya logrado un mayor grado de eficiencia en su utilización (Basu y Fernald, 2000).

La economía española parece que constituye un buen ejemplo con respecto a esta última afirmación. Los datos agregados muestran que la actual crisis económica ha determinado una notable mejora de la productividad del trabajo en contraste con el bajo pulso que esta variable mantuvo durante el período 1995-2007 , el cual se caracterizó, precisamente, por el logro de unas elevadas tasas de crecimiento y una capacidad de creación de empleo que parecían envidiables. Sin embargo, esto no coincide con lo que ha ocurrido en la mayor parte de los países avanzados ${ }^{2}$, lo cual parece que encuentra su mejor explicación en alguno de los rasgos que ha tenido y tiene la crisis en la economía española. En particular, la fuerte y rápida caída que ha registrado el empleo en España y la especialización de nuestra economía en actividades marcadamente influenciadas por el componente cíclico, como la construcción y algunos servicios de consumo final.

1 Durante el citado período 1995-2006/07 la productividad de la economía norteamericana registró fuertes ganancias, que -con limitadas excepciones-estuvieron siempre por encima de la mayor parte de las economías de la UE, tanto en términos de la productividad por trabajador como de la productividad multifactorial (MAROTO y CUADRADO, 2006; CUADRADO y MAROTO, 2012; O'MAHONY y VAN ARK, 2003; BBVA, 2010, entre otros). Durante ese mismo largo período expansivo la productividad laboral española se mantuvo, a su vez, claramente por debajo de la media de la UE-15 y, por supuesto, muy por debajo también de las tasas de la economía norteamericana. Además, la productividad multifactorial española, que nos informa sobre las mejoras en la eficiencia en cuanto al uso y organización de los factores, fue prácticamente nula-como media-e incluso negativa en alguno de los ejercicios del período indicado.

2 Tanto los trabajos teóricos y empíricos sobre el crecimiento de la productividad han llegado a un consenso en el que sólo unos pocos académicos se cuestionan el hecho de que la productividad agregada sea pro-cíclica. 
El principal objetivo de este artículo es, precisamente, profundizar en el comportamiento de la productividad en España desde la óptica del cambio cíclico. A estos efectos el punto de partida será una breve presentación de las diferencias que se observan al comparar el comportamiento de la tasa de variación del PIB, el empleo y la productividad en los países miembros de la UE-25 (sección 2), incluyendo a España por supuesto. A continuación, tras exponer los trazos básicos de la metodología que vamos a utilizar en nuestro análisis, ofreceremos los resultados que se obtienen al estudiar, a nivel agregado, la relación entre la productividad laboral y el ciclo en España (sección 3). El siguiente paso será presentar los resultados que ofrece el estudio de la evolución de la productividad por sectores, donde además profundizaremos en la coherencia y la sincronía cíclica de dicha productividad sectorial, así como en su volatilidad. Una breve nota final recogerá las principales conclusiones que se deducen del análisis realizado.

Los datos utilizados en nuestro estudio proceden de la base elaborada por The Conference Board (Total Economy Database, 2012), que permite abordar de forma solvente las comparaciones internacionales que se presentan en la sección 2, y de las cifras de la Contabilidad Nacional Trimestral, que elabora el INE (2012), utilizadas para desarrollar los análisis empíricos productividad-ciclo referidos al caso español.

\section{La crisis y su impacto en la productividad de la UE-25}

La profunda crisis económico-financiera iniciada en 2007 ha golpeado duramente a la mayor parte de los países de la Unión Europea, aunque con distintos grados de intensidad. Globalmente, y especialmente entre 2007 y 2009, el resultado de dicho impacto ha sido una desaceleración del ritmo de crecimiento de la productividad, tanto laboral como multifactorial, como consecuencia de la caída de la producción, la destrucción de empleo y, probablemente también, por la desaceleración de otros factores, como la calidad de la mano de obra o el uso del capital tecnológico.

Sin embargo, algunos países escapan a este comportamiento general. Entre ellos está España, que ha registrado tasas de crecimiento positivas de su productividad en el período 2007-2011 y que es, así, prácticamente el único país europeo donde la productividad aceleró su crecimiento con respecto al período 1995-2006. La causa que explica fundamentalmente esta mejora ha sido, sin duda, la fuerte destrucción de empleo que ha sufrido el país desde que se inició la crisis, sólo superada hasta 2010 por Irlanda y algunos países bálticos, como Estonia o Letonia, si bien en su conjunto alcanzan cifras muy inferiores a las españolas. 


\section{GRÁFICO 1 \\ IMPACTO DE LA CRISIS SOBRE LA PRODUCCIÓN, EMPLEO Y PRODUCTIVIDAD EN LA UNIÓN EUROPEA (EU-10 vs. EU-15)}

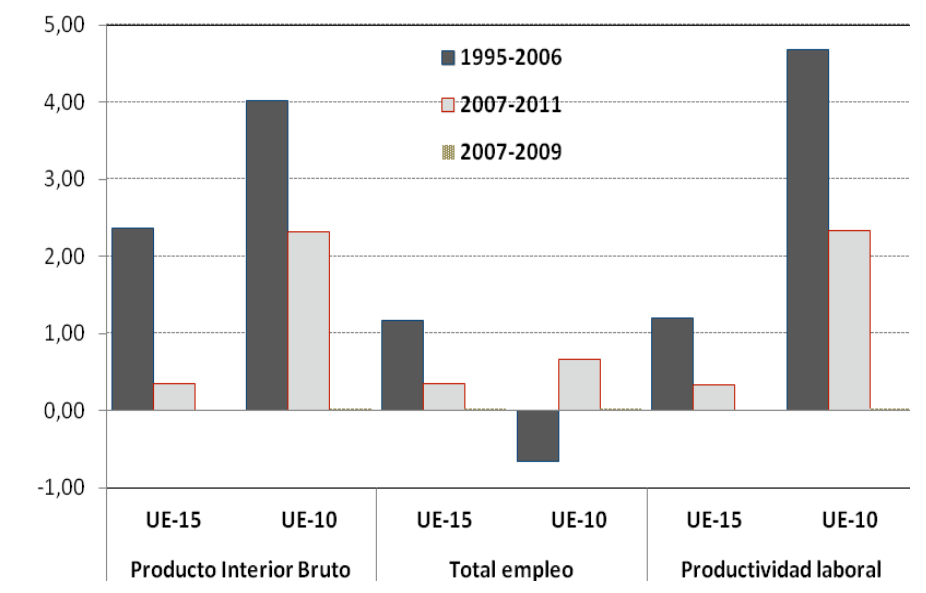

FUENTE: Elaboración propia, basado en datos de TCB (2012).

El Gráfico 1 muestra de forma clara que el efecto de la crisis en el contexto de la UE no ha sido homogéneo. Los nuevos Estados Miembros (UE-10) han registrado mayores tasas de crecimiento de su productividad durante el trienio 2007-2011 debido a que, a pesar de la fuerte caída de su producción bruta, las tasas de crecimiento de dicha variable están aún muy por encima de los países europeos más occidentales. Esto va unido a que, como consecuencia de que desde mediados de los años noventa la creación de empleo en la UE-10 ya era más baja, el impacto de la crisis no ha afectado tanto a su productividad como ha ocurrido en la mayoría de países de la UE-15. Lo cual ha dado lugar a un efecto estadístico que se ha traducido en unas tasas de crecimiento de su productividad más elevadas y siempre positivas (4,7 por 100 entre 1995 y 2006 y 2,3 por 100 entre 2007 y 2011; frente a los 1,2 por 100 y 0,3 por 100 de la UE-15). Como anteriormente se adelantó, durante el trienio 2007-2009 los resultados fueron claramente peores en toda Europa, observándose unas tasas medias de crecimiento anual prácticamente nulas en ambas zonas analizadas.

El Gráfico 2 permite observar mejor la heterogeneidad de comportamientos que ha tenido lugar en el contexto de la UE. Utilizando la metodología introducida por Camagni y Cappellin en 1985, que en anteriores trabajos sobre productividad ya habíamos empleado (Maroto y Cuadrado, 2009), pueden apreciarse cuáles fueron los países más dinámicos antes de la crisis y cómo han evolucionado sus respectivas posiciones a partir de 2007. Esta metodología distingue entre países dinámicos (primer cuadrante), países en restructuración (segundo cuadrante), países en retroceso (tercer cuadrante), y países intensivos en mano de obra (cuarto cuadrante). 


\section{GRÁFICO 2 \\ PRODUCCIÓN, EMPLEO Y PRODUCTIVIDAD EN EUROPA 1995-2006 \\ E IMPACTO DE LA CRISIS (2007-2011)}
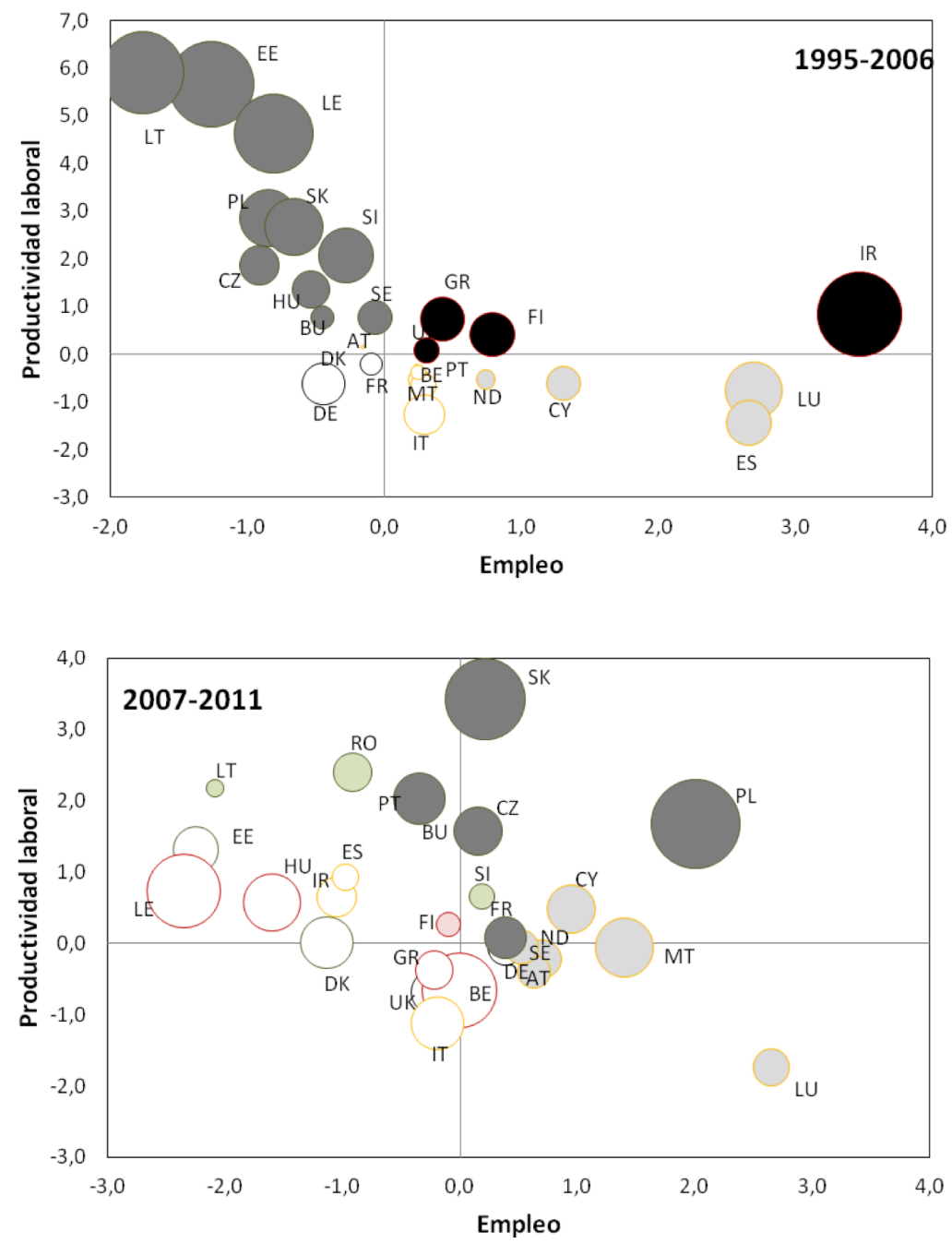

NOTAS: 1: Eje X: Diferencia entre crecimiento del empleo en cada país y la media europea (UE-25); Eje Y: Diferencia entre crecimiento de la productividad en cada país y la media europea; Tamaño de la burbuja: Diferencia entre crecimiento de la producción (valor añadido) en cada país y la media europea. 2: AT: Austria; BE: Bélgica; BU: Bulgaria; CY: Chipre; CZ: Chequia; DE: Alemania; DK: Dinamarca; EE: Estonia; ES: España; FI: Finlandia; FR: Francia; GR: Grecia; HU: Hungría; IR: Irlanda; IT: Italia; LE: Letonia; LT: Lituania; LU: Luxemburgo; MT: Malta; ND: Holanda; PO: Polonia; PT: Portugal; RO: Rumania; SI: Eslovenia; SK: Eslovaquia; SE: Suecia; UK: Reino Unido. 3: Tipologías de países: En negro países dinámicos (empleo y productividad por encima de la media); en blanco países en retroceso (empleo y productividad por debajo de la media); en gris oscuro países en restructuración de empleo (productividad por encima de la media y empleo por debajo); y en gris claro países intensivos en empleo (productividad por debajo de la media y empleo por encima).

FUENTE: Elaboración propia a partir de los datos de The Conference Board (TCB, 2012). 
En concreto se observa que en el período anterior a la crisis (1995-2006) los países europeos más dinámicos -con crecimientos superiores a la media europea, tanto en empleo como en productividad- fueron Irlanda, Grecia, Reino Unido, Finlandia y Letonia (en negro). Por el contrario, países como Alemania o Dinamarca (en blanco) presentaban menores tasas de crecimiento que la media europea, tanto en empleo como en productividad. Los restantes países comunitarios, o bien presentaban buenos resultados de productividad gracias a fuertes procesos de destrucción de empleo (en gris oscuro), como Suecia o la mayor parte de los países del este europeo, o bien no conseguían buenos resultados en términos de productividad por ser altamente intensivos en mano de obra, como ocurría en España (en gris claro).

Sin embargo, este panorama cambió radicalmente tras el comienzo de la crisis económico-financiera. Como muestra el gráfico inferior de la misma figura, la mayor parte de países que antes se situaban en los cuadrantes superiores -con buenos resultados de productividad-pasan a ocupar ahora posiciones en los cuadrantes inferiores.

Sólo algunos países del este de Europa, como Polonia, Chequia, Eslovaquia o Chipre, y ligeramente Francia y Holanda, se comportan dinámicamente. Por el contrario, un buen número de países, entre los que se cuentan Italia, Dinamarca, Eslovenia, Estonia o Hungría, estaban en retroceso. Más notorio es, si cabe, el caso de Letonia, Grecia y Finlandia, que a raíz de la crisis pasaron de ser dinámicos a estar en retroceso

El caso español, que analizaremos con mayor detalle en la próxima sección, muestra un cambio particularmente interesante. En el período 2007-2011 España ha conseguido situarse entre los países que registran mayores crecimientos de productividad, junto a Bulgaria o Eslovaquia, lo cual se debe únicamente -como ya se indicó- al fuerte proceso de destrucción de empleo que ha sufrido la economía española a partir del primero de dichos años, dado que el crecimiento de la producción bruta del país se ha situado por debajo de la media europea y la acumulación de capital productivo se ha mantenido a niveles muy bajos.

\section{Comportamiento cíclico de la productividad en España}

Como se adelantó en la sección anterior, la crisis ha tenido en España un perfil bastante diferente del de otros países europeos ${ }^{3}$. En nuestro caso, se han solapado claramente dos crisis: la de origen financiero y la vinculada a la profunda caída de la construcción de viviendas, que ha arrastrado a los numerosos sectores vinculados a este sector (Cuadrado et al., 2010). El impacto que ha supuesto el fin de la llamada «burbuja inmobiliaria» explica una gran parte de la caída del empleo, a la que también han contribuido la reducción del consumo familiar y, más recientemente, la contención del gasto y de las inversiones por parte del Estado, las comunidades autónomas y los municipios.

${ }^{3}$ Para un análisis más detallado de la evolución reciente de la productividad en nuestro país, véase CUADRADO y MAROTO (2012). 
En lo que a la productividad se refiere, lo que se observa claramente es que su comportamiento en España tiene una marcada influencia del ciclo económico, puesto que su evolución durante los últimos ciclos expansivo y recesivo ha sido significativamente diferente. Sin embargo, hay que preguntarse si la relación entre ciclo y productividad ha seguido los patrones del resto de economías cercanas, y si no es así por qué no lo ha hecho. En la sección anterior de este artículo se mostró que la productividad en la mayoría de los períodos y casos se comportaba de forma procíclica. Sin embargo, no ocurre lo mismo, al menos en estos últimos años, en el caso español. ¿Por qué?

El objetivo que pretende cubrir esta sección es doble. Por un lado, trataremos de demostrar mediante técnicas estadísticas que el comportamiento de la productividad española (productividad laboral ${ }^{4}$ ) está no sólo relacionado con el ciclo económico, sino que dicha relación es significativamente negativa. Y, por otro, vamos a tratar de ofrecer algunas posibles respuestas a la pregunta del por qué sucede esto, fundamentalmente desde el punto de vista de la estructura económica española y del comportamiento de las productividades sectoriales, que se analizará en la sección 4.

\subsection{Aspectos metodológicos}

Para relacionar la productividad con el ciclo económico es necesario clarificar, en primer lugar, dichas fluctuaciones cíclicas. A tal efecto es preciso descomponer en su tendencia y su componente cíclico tanto el crecimiento económico (aproximado a través del crecimiento del PIB nacional) como las variaciones de la productividad laboral. El ciclo económico se ha definido tradicionalmente como la secuencia de fases expansivas y contractivas de la actividad económica. En otras palabras, las recesiones y expansiones desde un punto de vista clásico se han aproximado por el signo negativo o positivo de la actividad económica. Sin embargo, en las últimas décadas también se han incluido dentro del término ciclo económico aquellas fases de ralentización en las que, aunque las tasas de crecimiento económico permanecen positivas, estas van decreciendo. Dado que en este trabajo nuestro interés se centra en el comportamiento cíclico de la productividad, aquí adoptaremos este último concepto más amplio.

$\mathrm{Al}$ analizar las fluctuaciones cíclicas es necesario estimar en las series de productividad la tendencia subyacente de cada serie analizada -en nuestro caso la productividad- con objeto de establecer el componente estructural, o a largo plazo, de aquellas desviaciones a corto plazo, o cíclicas. Para llevar a cabo esta tarea existen varios métodos. Zarnowitz y Ozyldirim (2001) compararon y contrastaron algunos de ellos y alcanzaron la conclusión de que todos obtienen resultados muy próximos.

${ }^{4}$ En el análisis solamente se tomará como referencia la productividad laboral ya que para poder analizar adecuadamente el comportamiento de la PMF en la economía española sería preciso disponer de información trimestral muy detallada, que de momento no existe para algunas de las variables que sería obligado considerar. 
El filtro introducido por Hodrick y Prescott en 1997 (a partir de ahora HP) es uno de los métodos que obtienen una correcta descomposición de la tendencia y la fluctuación cíclica y es el que vamos a utilizar en este caso.

Dicho filtro ${ }^{5}$, basado en la definición original de Lucas (1977) sobre el componente cíclico de una variable como aquellas desviaciones de la tendencia suavizada de dicha variable, estima un componente tendencial minimizando las desviaciones de dicha tendencia. Se trata de un problema de optimización sujeto a restricciones, donde la restricción es el parámetro de suavización, generalmente denominado $\lambda$. La expresión formal del problema es la siguiente:

$$
\min \left\{\sum_{t=1}^{N}\left(Y_{t}-T_{t}\right)^{2}+\lambda \sum_{t=k+1}^{N}\left(\nabla^{k} T_{t}\right)^{2}\right\}
$$

donde $Y_{t}$ representa el valor de la serie original, $T_{t}$ la tendencia y $\nabla T_{t}=T_{t}-T_{t-1}$, $\nabla^{k}=\nabla\left(\nabla^{k-1}\right)$ el operador de retardos para un determinado parámetro $\lambda$.

El primer componente de la ecuación anterior aproxima la bondad de ajuste en la minimización, mientras que el segundo mide el grado de suavización, penalizando las deceleraciones en el crecimiento del componente de tendencia. Variaciones en el parámetro $\lambda$ alteran el equilibrio entre ambos componentes de la ecuación. La formulación que hemos presentado muestra que si lambda tiende a cero, las desviaciones cíclicas se minimizan sin restricciones, por lo que la tendencia será igual a la serie original. Igualmente, si el parámetro tiende a infinito, la tendencia converge a una serie lineal.

La principal razón para elegir el filtro $\mathrm{HP}^{6}$ ha sido que en varios trabajos recientes sobre comportamiento cíclico de la productividad fue el que se utilizó y su empleo ofrece, por tanto, una correcta vía para comparar nuestros resultados con los concluidos en otros trabajos, como los de Inklaar y McGuckin (2003), Maroto y Rubalcaba (2008) para los países de la Unión Europea, Gordon (2003) para EE UU, o Cuadrado y Ortiz (2001) y Maroto (2011) para el caso español. Otra de las ventajas de dicho filtro es que ofrece estimaciones incluso para el final de la muestra. Aunque dichas estimaciones tienen menos robustez que aquellas para períodos intermedios ${ }^{7}$,

${ }^{5}$ Véase KING y REBELO (1993) y BLACKBURN y RAVN (1991) para un estudio detallado sobre el citado filtro.

${ }^{6}$ En lugar de otros posibles filtros, como el de KALMAN (1960) u otros filtros Butterworth, que permiten distintas formas estructurales en el término cíclico de las series. Véase WELCH y BISHOP (1995), HARVEY y TRIMBUR (2001) o FRENCH (2005), entre otros, para una introducción a estas aproximaciones metodológicas.

${ }^{7}$ Los puntos (años en nuestro caso) finales de la muestra son siempre motivo de debate en la metodología sobre filtros de tendencia. La razón es que normalmente utilizan medias móviles para suavizar las series por lo que necesitan tanto valores pasados como futuros de la series para la estimación de los valores intermedios. Aunque la mayoría de métodos obtienen resultados muy similares para los períodos intermedios, no ocurre lo mismo para los años finales de la muestra. Dada la importancia de la recesión observada al final del periodo de análisis, el filtro HP ofrece una óptima elección metodológica para este trabajo. Sin embargo, existen experimentos que demuestran que los resultados no cambiarían significativamente si se utilizasen otros filtros. 
en nuestro caso son las más interesantes debido a que explican el comportamiento cíclico en el período de crisis económico-financiera.

La puesta en práctica de cualquier filtro de tendencia requiere determinar el valor del parámetro lambda. Hodrick y Prescott (1997) sugirieron en su trabajo seminal que dicho parámetro debería ser igual a 1600 para datos trimestrales. Por su parte, Ravn y Uhlig (2002) sugieren una simple fórmula para encontrar el parámetro para diferentes frecuencias de los datos. Como en nuestro caso la frecuencia es trimestral, el parámetro lambda de Ravn-Uhlig coincide con el de Hodrick-Prescott y, por tanto, será igual a 1600.

A pesar de las ventajas hasta ahora apuntadas, las propiedades estadísticas de los componentes filtrados, o sin tendencia, siguen siendo objeto de debate ${ }^{8}$. Por esta razón, posteriormente se analizará la sensibilidad de nuestras estimaciones a través del cálculo de medidas de volatilidad y de sincronía cíclica. Para ello, la desviación estándar de las series filtradas se utiliza como aproximación de la volatilidad del componente cíclico en términos relativos. Posteriormente, se calculan coeficientes de correlación entre la productividad y el crecimiento económico con objeto de analizar si la productividad -tanto agregada como sectorial- se comporta pro-cíclica o contra-cíclicamente, y la sincronía con respecto al ciclo general de dichas variables. En concreto, un valor positivo (negativo) y estadísticamente significativo indicará que la productividad es pro-cíclica (contra-cíclica), mientras que valores cercanos a cero indicarán una no-correlación periódica entre ambas variables. Para los datos trimestrales usamos un punto de corte de 0,35 -aproximando los valores que rechazan la hipótesis de que la correlación sea nula al 5 por 100 de significación en una distribución $t$ de dos colas ${ }^{9}$. Adicionalmente, si el coeficiente de correlación presente su valor máximo (en términos absolutos) en el período $t-i, t$ o $t+j$, diremos que el ciclo está adelantado por $i$ períodos, coincidente, o retrasado $j$ períodos con respecto al ciclo general, respectivamente.

\subsection{Resultados obtenidos a nivel agregado}

Varios trabajos han demostrado en las últimas décadas la correlación que existe, a nivel agregado ${ }^{10}$, entre la productividad laboral y el ciclo económico, basado en diferentes medidas de la producción final. Todos ellos han concluido que la productividad laboral tiene un comportamiento pro-cíclico. Sin embargo, esto no evita que a nivel sectorial o en el caso de algunos países concretos, el comportamiento de la productividad sea a-cíclico o incluso contra-cíclico (Maroto, 2010). En concreto, en el caso español parece claro que, al menos desde mediados de los años noventa,

\footnotetext{
8 Véanse, entre otros, KING y REBELO (1993), COGLEY (1990) o HARVEY y JAGER (1991).

9 En la línea de otros trabajos relevantes, como ROSENTHAL (1991), KIM et al. (2003), DIMELIS (2001), FIORITO y KOLLINTZAS (1994), CHRISTODOULAKIS et al. (1995) o MAROTO (2010), entre otros.

${ }_{10}$ Véanse, entre otros, KYDLAND y PRESCOTT (1982), HANSEN (1985), PRESCOTT (1986), MCCALLUM (1989), BENHABIB et al. (1991), HANSEN y WRIGHT (1992) y BENCIVENGA (1992).
} 


\section{CUADRO 1 \\ CRECIMIENTO* EN LA PRODUCCIÓN, EMPLEO Y PRODUCTIVIDAD EN ESPAÑ $A$}

(Serie real, corregida, tendencia y ciclo)

\begin{tabular}{|l|c|c|c|c|c|}
\hline & Producción & Empleo & $\begin{array}{r}\text { Productividad } \\
\text { por trabajador }\end{array}$ & Horas & $\begin{array}{c}\text { Productividad } \\
\text { por hora }\end{array}$ \\
\hline \multicolumn{7}{|c|}{$1995-2011$} \\
\hline Real & 1,09 & 0,41 & 0,67 & 0,20 & 0,63 \\
\hline Corregida & 1,12 & 0,43 & 0,69 & 0,24 & 0,65 \\
\hline Tendencia & 1,15 & 0,45 & 0,70 & 0,25 & 0,65 \\
\hline Ciclo & $-0,03$ & $-0,02$ & $-0,01$ & $-0,01$ & 0,00 \\
\hline \multicolumn{7}{|c|}{$1995-2006$} \\
\hline Real & 1,60 & 0,79 & 0,81 & 0,54 & 1,02 \\
\hline Corregida & 1,54 & 0,79 & 0,75 & 0,64 & 0,83 \\
\hline Tendencia & 1,52 & 0,73 & 0,79 & 0,58 & 0,82 \\
\hline Ciclo & 0,02 & 0,06 & $-0,04$ & 0,06 & 0,01 \\
\hline \multicolumn{7}{|c|}{$2007-2011$} \\
\hline Real & $-0,35$ & $-0,64$ & 0,29 & $-0,35$ & 0,01 \\
\hline Corregida & $-0,03$ & $-0,55$ & 0,52 & $-0,39$ & 0,35 \\
\hline Tendencia & 0,11 & $-0,35$ & 0,45 & $-0,27$ & 0,38 \\
\hline Ciclo & $-0,14$ & $-0,20$ & 0,06 & $-0,11$ & $-0,03$ \\
\hline
\end{tabular}

NOTA: * Tasas de crecimiento con respecto al trimestre anterior.

FUENTE: Elaboración propia basada en Contabilidad Nacional Trimestral (INE, 2012).

la productividad laboral ha sido contra-cíclica a nivel agregado. Pasemos a demostrar estadísticamente este hecho y también a intentar aportar posteriormente algunas explicaciones -desde el punto de vista sectorial- sobre por qué el caso español se diferencia de la mayoría de casos estudiados en la literatura especializada.

El Cuadro 1 muestra los resultados básicos del análisis de descomposición que anteriormente se ha descrito (tendencia y fluctuación cíclica). Los datos revelan las tasas de crecimiento de la producción, el empleo y la productividad, tanto reales como corregidas por estacionalidad y calendario ${ }^{11}$, así como las de los componentes de tendencia y de carácter cíclico. Los resultados no sólo se muestran para la totalidad del período 1995-2011, sino diferenciando también dos sub-períodos: pre-crisis o expansivo (1995-2006) y de crisis o recesivo (2007-2011).

La principal conclusión que cabe extraer de los datos del Cuadro 1 es el poco peso que tiene, en general, el componente cíclico, unido al comportamiento opuesto que han experimentado, por un lado, la producción y el empleo y, por otro, la productividad, durante los años transcurridos desde el inicio de la crisis hasta la fecha. En con-

${ }^{11}$ Series ofrecidas directamente por el INE en su Contabilidad Nacional Trimestral. 
creto, se observa que el componente cíclico para la totalidad del período 2007-2011 supone únicamente el 2,3 por $100,3,1$ por 100 y 0,5 por 100 del crecimiento real corregido de las cinco variables analizadas. Sin embargo, resulta evidente que durante el período de crisis el efecto del ciclo sobre el crecimiento de dichas variables ha sido mucho más significativo. En concreto, el comportamiento cíclico ha contabilizado el 450 por 100 en el caso del PIB nacional, mientras que los porcentajes en el caso del empleo y productividad han sido, respectivamente, el 37, 30, 13 y 7 por 100 .

Hasta ahora se ha analizado la evolución real y de tendencia junto al componente cíclico. Sin embargo, puesto que nuestro objetivo es el análisis del ciclo y la productividad, el Gráfico 3 muestra la evolución del componente cíclico de la producción, el empleo y la productividad en la economía española desde mediados de los años noventa hasta comienzos del año 2011. Dicha comparación muestra varios hechos interesantes que conviene resaltar.

Desde el punto de vista general, dos son las conclusiones que se obtienen de los datos:

- La primera es que la reducción en la volatilidad del ciclo español ${ }^{12}$ que venía observándose desde los años 50s (Cuadrado y Ortiz, 2001), ha continuado hasta mediados de la primera década del siglo XXI. Sin embargo, a partir del año 2006 dicha volatilidad ha sufrido un significativo cambio, aumentando notoriamente la volatilidad del ciclo español durante el período de crisis.

- Y, en segundo lugar, los períodos vividos durante fases expansivas han tenido una duración mayor que aquellos en los que la economía española se ha visto inmersa en recesiones. Aunque el período analizado en el Gráfico 3 no contiene más que un ejemplo de cada tipo, varios estudios recientes realizados por la Fundación BBVA parecen indicar que la duración del ciclo recesivo actual no alcanzará en ningún caso la que tuvo el ciclo expansivo que finalizó en 2007.

${ }^{12}$ Fenómeno que no sólo ha podido observarse en el caso español, sino también en otros países, fundamentalmente aquellos trabajos realizados a cabo del National Bureau of Economic Research (NBER). 


\section{GRÁFICO 3 \\ COMPARACIÓN DEL CICLO ESPAÑOL. PRODUCCIÓN, EMPLEO Y PRODUCTIVIDAD}

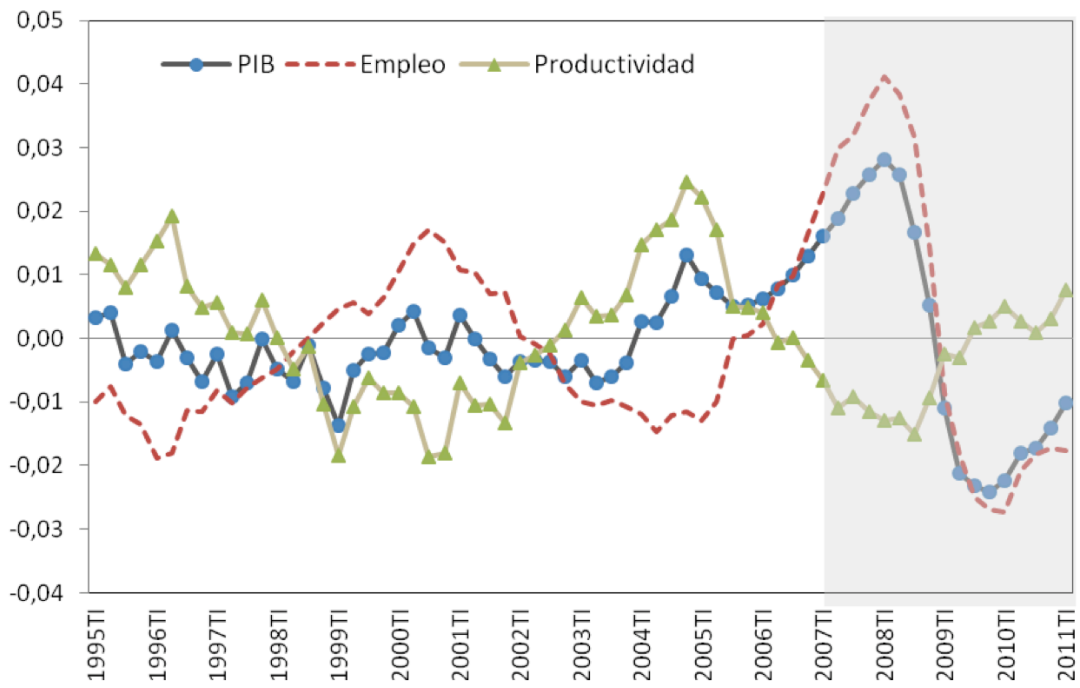

FUENTE: Elaboración propia basada en Contabilidad Nacional Trimestral (INE, 2012).

Por otra parte, el Gráfico 3 nos muestra también las primeras conclusiones acerca de la relación existente entre los ciclos de la productividad española y los ciclos de la producción y empleo. A destacar, en particular, lo siguiente:

i) La relación entre el crecimiento económico y el crecimiento del empleo -tanto en número de trabajadores como en horas trabajadas ${ }^{13}$ - registra un notable cambio entre los dos períodos seleccionados. Aunque la relación entre ambos componentes cíclicos es positiva para la totalidad del período 1995-2011 (con un coeficiente de correlación igual a 0,77 para el caso del número de trabajadores y de 0,89 para el caso de las horas trabajadas, con $p$-valores de 0,00 en ambas ocasiones), dicha relación pro-cíclica del empleo viene forzada por el período de crisis (donde el coeficiente de correlación asciende a 0,99 y 0,98 respectivamente, con $p$-valores de 0,00). En el período entre 1995 y 2006 la relación, aunque positiva, no fue significativa.

ii) Tanto a nivel de productividad por trabajador como de productividad por hora trabajada, la relación con el ciclo económico es ligeramente negativa para la totalidad del período $(-0,11$ y $-0,06$, respectivamente). Sin embargo, este comportamiento contra-cíclico de la productividad española es mucho más significativo en el período

13 Donde la disponibilidad de datos es menor ya que la Contabilidad Nacional Trimestral del INE únicamente ofrece datos sobre las horas trabajadas a partir del primer trimestre del año 2000. 
reciente de crisis, cuando los coeficientes de correlación han sido, respectivamente, de $-0,89$ y $-0,52$ con $p$-valores de 0,00 y 0,02$)$. Por el contrario, antes de esta crisis, la relación era ligeramente pro-cíclica (con coeficientes de correlación en torno a 0,5 )

\section{Análisis sectorial: resultados más relevantes y el problema de la volatilidad}

En la sección anterior se han presentado algunos hechos estilizados sobre el comportamiento cíclico de la productividad española a nivel agregado. La principal conclusión que se extrae es que, al contrario de lo sucedido en otros países y fases cíclicas, en los últimos años la productividad ha tenido en España un comportamiento significativamente contra-cíclico. Sin embargo, cabe presumir que dicho comportamiento podría estar influenciado, entre otras variables que aquí no analizaremos, por la estructura sectorial de la economía española y por las posibles respuestas diferenciadas de los distintos sectores económicos ante el ciclo económico. Particularmente en el caso de aquellos cuyo peso en el conjunto del tejido productivo económico es más importante, como sucede con algunas actividades de servicios y manufacturas. Esto justifica que analicemos ahora el comportamiento cíclico de la productividad española desde un punto de vista sectorial.

Al igual que para el conjunto de la economía, la tendencia y el componente cíclico se han obtenido filtrando las series reales corregidas a través del filtro HP. En consecuencia, el ciclo será la diferencia entre la serie corregida expresada en logaritmos y la tendencia extraída a través del citado filtro. Los resultados obtenidos para el VAB sectorial y la productividad sectorial se sintetizan en los Gráficos 4a y 4b. Aunque los cálculos se han realizado para todos los sectores, los gráficos incluidos sólo muestran la evolución del ciclo económico general y el componente cíclico de las manufacturas, la construcción y los servicios.

A partir de las estimaciones efectuadas pueden extraerse algunas conclusiones iniciales desde el punto de vista sectorial:

- En cuanto al ciclo de la producción (Gráfico 4a), durante el período 1995-2011 todos los sectores analizados tienen un comportamiento pro-cíclico. Los coeficientes de correlación son, respectivamente, iguales a $0,78,0,74$ y 0,87 . Sin embargo, comportamiento se debe principalmente a la correlación fuertemente positiva que se observa desde el año 2007 entre los componentes cíclicos sectoriales y el ciclo general (con coeficientes iguales a $0,86,0,90$ y 0,96 respectivamente).

- En cuanto al ciclo de la productividad laboral (Gráfico 4b), aunque también se observan correlaciones positivas entre los ciclos sectoriales y el general, este comportamiento pro-cíclico (con coeficientes de correlación iguales a $0,54,0,72$ y 0,85) es menos significativo que en el caso del VAB. Adicionalmente, durante el período de crisis esta relación positiva es, si cabe, menos fuerte que en el período expansivo anterior (coeficientes de correlación igual a 0,20,0,83 y 0,64 respectivamente). 


\section{GRÁFICO 4A \\ COMPARACIÓN DEL CICLO ECONÓMICO POR SECTORES EN ESPAÑA. VAB SECTORIAL, 1995-2011}

a) Manufacturas

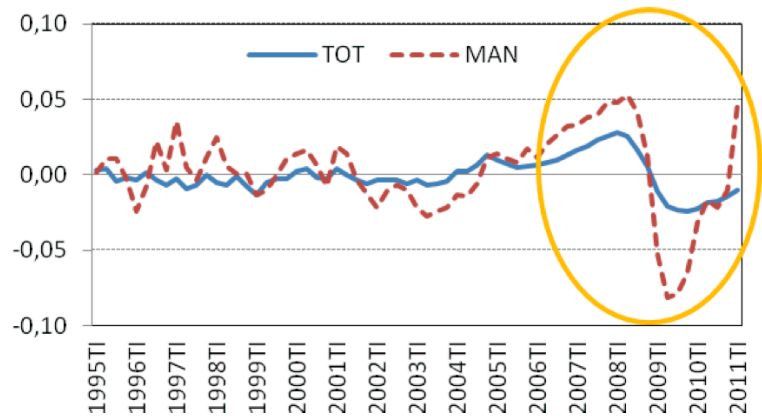

b) Construcción

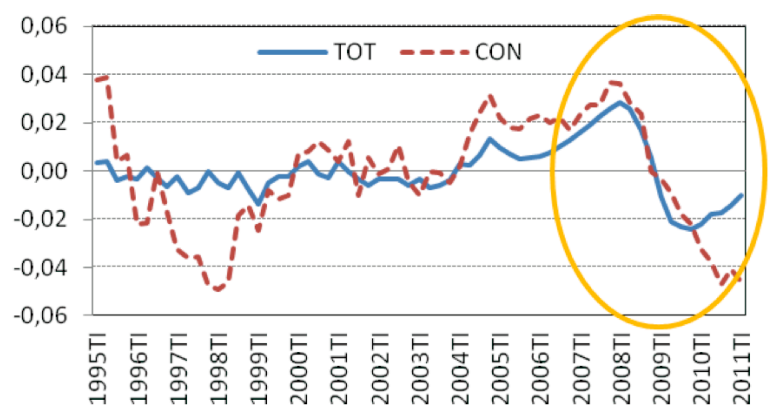

c) Servicios

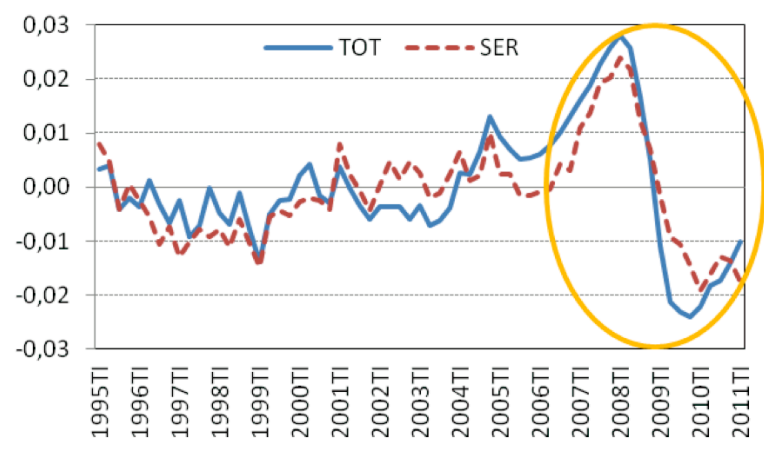

FUENTE: Elaboración propia basada en Contabilidad Nacional Trimestral (INE, 2012). 


\section{GRÁFICO 4B \\ COMPARACIÓN DEL CICLO ECONÓMICO POR SECTORES EN ESPAÑA. \\ PRODUCTIVIDAD SECTORIAL, 1995-2011}

a) Manufacturas

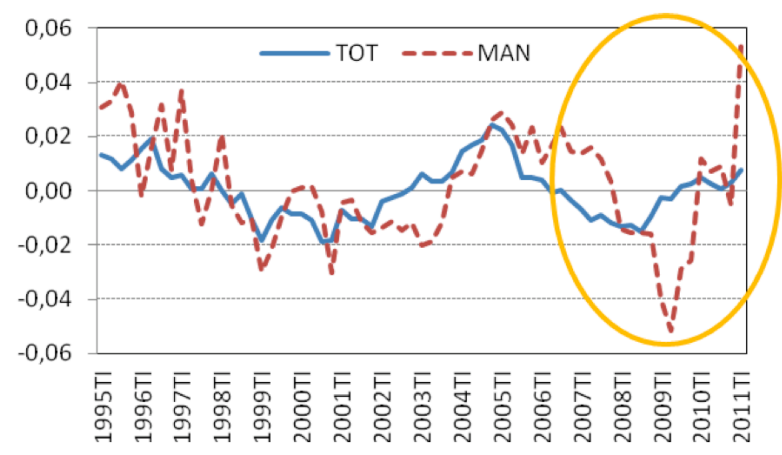

b) Construcción

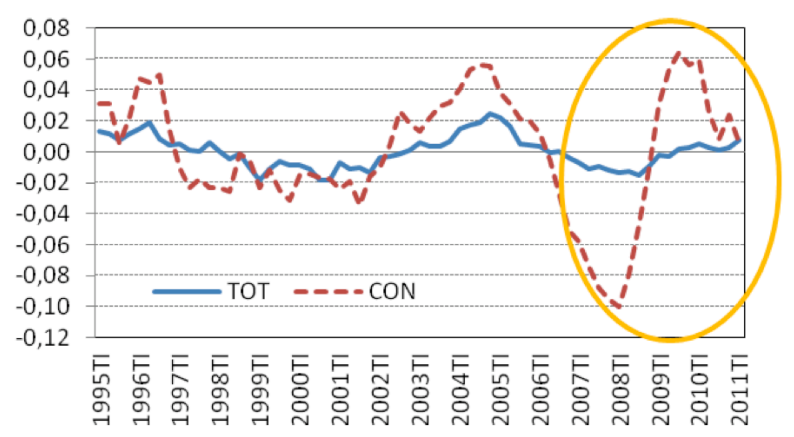

c) Servicios

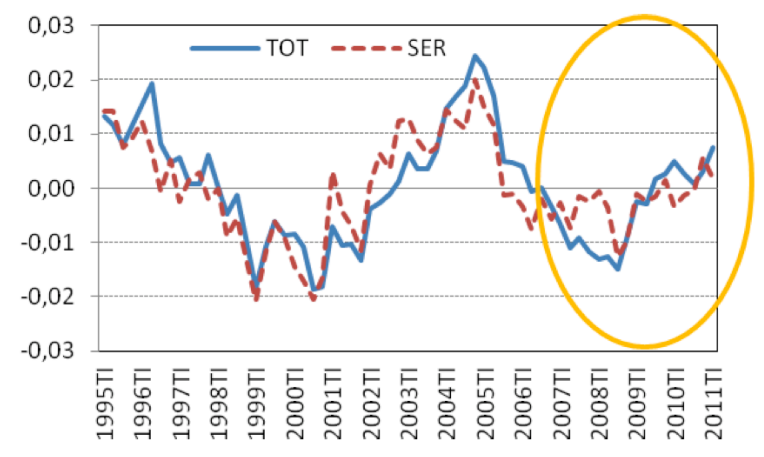

FUENTE: Elaboración propia basada en Contabilidad Nacional Trimestral (INE, 2012). 
Por otra parte, en los trabajos más recientes sobre ciclos es común analizar el comportamiento de los mismos a través de algunos estadísticos que puedan ofrecer una mejor comprensión de dichos patrones que el análisis gráfico y las correlaciones anteriormente presentadas. Por este motivo, pueden calcularse la volatilidad (medida como la desviación típica de las fluctuaciones cíclicas de la variable analizada) y la volatilidad relativa (medida como el cociente entre la volatilidad de la variable de referencia - generalmente el PIB - y la volatilidad del resto de variables analizadas). Ambas nos ofrecen una imagen más clara de la magnitud de las fluctuaciones cíclicas, así como sobre su relación con el ciclo general. En el caso del análisis sectorial que estamos llevando a cabo en este sub-apartado, estos indicadores parecen ser de obligada observación.

El Cuadro 2 muestra los cálculos sobre las volatilidades de la productividad sectorial en España durante el período 1995-2011. Para su mejor comprensión, dicho período se ha dividido, siguiendo la línea de todo el trabajo, en dos sub-períodos. El primero, caracterizado por ser un período expansivo (1995-2006), tiene una mayor relatividad, tanto para el conjunto de la economía $(1,1)$ como en la agricultura $(4,6$ $v s .3,9)$ y el sector servicios $(0,9 v s .0,6)$. Sin embargo, el sub-período dominado por la crisis tiene una menor volatilidad general $(0,7)$, aunque a nivel sectorial se observan mayores volatilidades relativas tanto en las manufacturas $(3,4 v s .1,7)$ como en la construcción $(8,2$ vs. 2,6) y la energía $(5,7$ vs. 2,4$)$.

\section{CUADRO 2}

VOLATILIDAD CÍCLICA DE LA PRODUCTIVIDAD SECTORIAL EN ESPAÑA

\begin{tabular}{|l|c|c|c|c|c|c|}
\hline & Total & $\begin{array}{c}\text { Agricul- } \\
\text { tura }\end{array}$ & Energía & $\begin{array}{c}\text { Manufac- } \\
\text { turas }\end{array}$ & $\begin{array}{c}\text { Construc- } \\
\text { ción }\end{array}$ & Servicios \\
\hline \multicolumn{7}{|c|}{$1995-2011$} \\
\hline Volatilidad & 1,04 & 4,55 & 3,07 & 2,06 & 3,94 & 0,93 \\
\hline Volatilidad real & 1,00 & 4,39 & 2,96 & 1,99 & 3,80 & 0,89 \\
\hline \multicolumn{7}{|c|}{$1995-2006$} \\
\hline Volatilidad & 1,09 & 4,98 & 2,62 & 1,85 & 2,83 & 1,04 \\
\hline Volatilidad real & 1,00 & 4,57 & 2,41 & 1,70 & 2,59 & 0,95 \\
\hline \multicolumn{7}{|c|}{$2007-2011$} \\
\hline Volatilidad & 0,73 & 2,86 & 4,19 & 2,50 & 5,99 & 0,43 \\
\hline Volatilidad real & 1,00 & 3,91 & 5,72 & 3,42 & 8,19 & 0,59 \\
\hline
\end{tabular}

FUENTE: Elaboración propia basada en Contabilidad Nacional Trimestral (INE, 2012). 
Tanto desde el punto de vista general como sectorial, los indicadores mostrados en el Cuadro 2 confirman el comportamiento cíclico anteriormente descrito para los principales sectores de actividad, ya que, con la única excepción del sector servicios -fundamentalmente impulsada por los servicios de mercado, ya que los servicios de no-mercado tienen un comportamiento cíclico completamente diferente-, los demás sectores muestran mayor volatilidad que el conjunto de la economía. Este hecho coincide con los resultados de otros trabajos sobre el ciclo español, como Cuadrado y Ortiz (2001) o Maroto (2011).

Para complementar el análisis realizado, hemos realizado un estudio sobre la sincronía cíclica entre la productividad sectorial y el ciclo económico, con el fin de contrastar la hipótesis del posible comportamiento contra-cíclico de la productividad española. Para ello se han estimado coeficientes de correlación entre distintos períodos de tiempo, adelantados, coincidentes y retrasados, de la variable bajo estudio y la variable de referencia (que en nuestro caso será el ciclo económico general o fluctuación cíclica del PIB nacional). Este ejercicio refleja el nivel de coherencia o de sincronía entre sus ciclos. A tal efecto consideraremos que la productividad es pro-cíclica si sus fluctuaciones sigan la misma dirección que el ciclo económico -y por tanto, que muestren una correlación positiva-. Si las fluctuaciones entre ambas variables son opuestas -cuando el ciclo es expansivo, el ciclo de la variable se contrae, y viceversa- la productividad será contra-cíclica. Por último, si no existe relación alguna entre ambas se calificará la productividad como a-cíclica.

Por otra parte, cabe tener en cuenta, asimismo, que la productividad -tanto agregada como sectorial- puede anticiparse, coincidir o retrasarse en su ciclo con respecto al ciclo económico general. Se anticipará cuando las subidas (bajadas) ocurran antes que en la producción económica. Por el contrario, si dichas subidas (bajadas) ocurren como resultado de fluctuaciones en la actividad económica, se dirá que la productividad es retrasada. Finalmente, si las variaciones tienen lugar en el mismo período de tiempo, entonces las variables serán coincidentes.

Para analizar tanto la coherencia como la sincronía se calculan los coeficientes de correlación $\rho_{j}$ entre los diferentes periodos retrasados y adelantados, hasta un total de cinco períodos $(j \in 0,1, \ldots, 5)$. Consideraremos que la productividad es pro-cíclica (contra-cíclica) si el mayor coeficiente de correlación es positivo (negativo) y mayor que 0,5 (en valor absoluto). Cuando el mayor coeficiente de correlación sea menor que 0,5 podrá concluirse que la productividad es a-cíclica. Por otra parte, cabe estudiar también si el ciclo de la productividad es adelantado, coincidente o retrasado con relación al ciclo económico general, lo que podrá deducirse cuando el coeficiente de correlación máximo se encuentre, respectivamente, para $j>0, j=0$ o $j<0$. 


\section{CUADRO 3}

\section{COHERENCIA Y SINCRONÍA CÍCLICA DE LA PRODUCTIVIDAD SECTORIAL EN ESPAÑA}

\begin{tabular}{|l|c|c|c|c|c|c|}
\hline & Total & $\begin{array}{c}\text { Agri- } \\
\text { cultura }\end{array}$ & Energía & $\begin{array}{c}\text { Manu- } \\
\text { facturas }\end{array}$ & $\begin{array}{c}\text { Construc- } \\
\text { ción }\end{array}$ & Servicios \\
\hline \multicolumn{7}{|c|}{ 1995-2011 } \\
\hline Coherencia & Acíclica (-) & Acíclica (-) & Acíclica (-) & Procíclica & Contracíclica & Acíclica (+) \\
\hline Sincronía & Adelantada (2) & Retrasada (5) & Retrasada (4) & Retrasada (3) & Coincidente & Adelantada (4) \\
\hline \multicolumn{7}{|c|}{$1995-2006$} \\
\hline Coherencia & Acíclica (+) & Acíclica (-) & Acíclica (+) & Procíclica & Acíclica (+) & Acíclica (+) \\
\hline Sincronía & Coincidente & Retrasada (5) & Adelantada (5) & Adelantada (1) & Retrasada (5) & Coincidente \\
\hline \multicolumn{7}{|c|}{$2007-2011$} \\
\hline Coherencia & Contracíclica & Procíclica & Acíclica (+) & Procíclica & Contracíclica & Procíclica \\
\hline Sincronía & Adelantada (1) & Adelantada (5) & Adelantada (1) & Retrasada (4) & Coincidente & Adelantada (3) \\
\hline
\end{tabular}

NOTA: Entre paréntesis el signo de la correlación en el caso de la coherencia, y el número de retardos en el caso de la sincronía.

FUENTE: Elaboración propia basada en Contabilidad Nacional Trimestral (INE, 2012).

El Cuadro 3 muestra los principales resultados obtenidos, tanto en relación con la coherencia como en cuanto a la sincronía cíclica de la productividad sectorial en España, siempre con referencia al conjunto del período analizado en este trabajo. Lo que muestran los resultados es que, en términos agregados, la productividad española presenta -como ya se ha venido subrayando- un comportamiento notoriamente diferenciado antes y después del comienzo de la crisis. Mientras que entre 1995 y 2006 el patrón era $a$-cíclico, aunque con una relación positiva y coincidente, en los últimos años, dicha relación se ha vuelto contra-cíclica (y estadísticamente significativa) y ligeramente adelantada (un período). Debido a este hecho, la productividad laboral española ha experimentado el crecimiento que ya se expuso y explicó en la sección anterior, mientras que la actividad económica general sufría una notable recesión, como el resto de economías europeas de nuestro entorno, aunque más extensa en el tiempo que muchas de ellas.

A nivel sectorial se observan comportamientos cíclicos bastante bien diferenciados. Por una parte, el sector de la Construcción presenta un patrón muy parecido al del total de la economía, ya que el signo de su relación con el signo de la economía ha pasado de positivo a negativo durante la crisis. El caso de la Agricultura es, sin embargo, el opuesto. Y, por último, tanto los Servicios como las Manufacturas presentan un patrón diferente al del total de la economía. Con anterioridad a la crisis, la productividad de estos dos sectores era a-cíclica, aunque con signo positivo, mientras que durante la misma esta relación no ha sufrido cambios, aunque sí se ha intensificado la correlación positiva con el ciclo general de la economía española. 


\section{GRÁFICO 5 \\ VOLATILIDAD Y PERSISTENCIA DE LA PRODUCTIVIDAD EN ESPAÑA (1995-2011)}
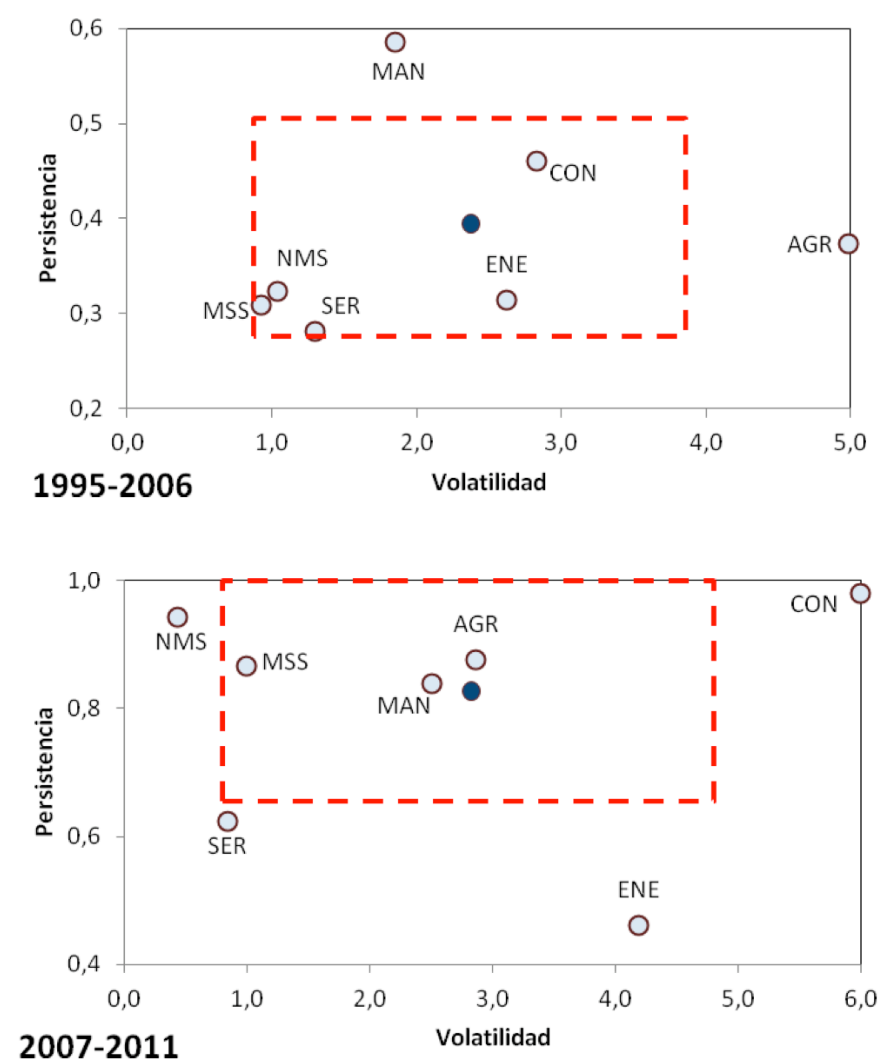

FUENTE: Elaboración propia basada en Contabilidad Nacional Trimestral (INE, 2012).

Para concluir nuestro análisis del comportamiento cíclico de la productividad española, el Gráfico 5 resume la información presentada hasta ahora. En particular, se muestran conjuntamente la volatilidad (medida a través de la desviación típica de las fluctuaciones cíclicas) y la persistencia (medida a través del coeficiente de correlación que delimitaba la coherencia anteriormente explicada en el Cuadro 3) de la productividad sectorial en nuestro país para los dos sub-períodos analizados en todo este trabajo.

El rectángulo discontinuo describe el área que delimita una desviación típica respecto a la media sin ponderar de todos los valores sectoriales. La teoría clásica sugeriría que la mayoría de sectores deberían encontrarse en el interior o alrededor de dicho rectángulo. En el período que precedió a la crisis, las únicas excepciones 
fueron las manufacturas y la agricultura, mientras que en el período más reciente, ya bajo la crisis económico-financiera, sólo el sector energía es la excepción El resto de sectores sugieren que las fluctuaciones cíclicas de la productividad sectorial en España tienen una duración e intensidad similar a la agregada, aunque las intensidades que se observan durante el período de crisis (Gráfico 5 inferior) son ligeramente más altas que en el período expansivo anterior.

\section{Notas finales a destacar}

El tema tratado en este artículo no se cierra, por supuesto, con los resultados alcanzados. De hecho, quedan abiertas varias líneas de trabajo para futuras investigaciones. Sin embargo, los análisis que han servido de base para elaborar este artículo ofrecen algunos resultados que cabe destacar como cierre del mismo.

Un primer aspecto a subrayar es que, como ya se sabía pero como aquí se ha comprobado de nuevo, las fluctuaciones cíclicas que sufren las economías afectan claramente a la productividad, lo cual implica que los cambios que puede mostrar la evolución de esta variable deben analizarse siempre teniendo muy en cuenta los efectos del ciclo. El caso de España es, en este sentido paradigmático. La grave crisis económico-financiera que se inició en 2007 ha tenido un gran impacto en la economía española, sobre todo en cuanto a la destrucción de empleos, lo que se ha convertido en el principal factor explicativo de los incrementos en la productividad laboral que se han producido desde 2008 hasta 2011. Los datos de avance sobre 2012 apuntan a que este giro ha continuado, sin que pueda atribuirse a una intensificación del capital ni, muy probablemente, a una intensa introducción de nuevas tecnologías. La caída del empleo es el principal factor explicativo, al que se unen cambios organizativos en las empresas cuyos efectos son todavía difíciles de evaluar.

El estudio realizado permite poner de relieve otros aspectos interesantes sobre la relación entre productividad y ciclo. El primero de ellos es que a diferencia de lo que ha ocurrido y suele ocurrir en bastantes países, en el sentido del carácter pro-cíclico que muestra la evolución de la productividad, en el caso español esta variable se comporta contra-cíclicamente. Ocurrió ya así en la fase más expansiva de nuestra economía (1995-2006), con tasas de variación de la productividad muy bajas, y ha ocurrido a partir del inicio de la crisis, con tasas de variación anual por encima o muy próximas al 2 por 100. La principal explicación hay que buscarla en el tipo de empleos creados en el período expansivo -en sectores con muy baja productividad- y en la destrucción generalizada de empleo que ha tenido y tiene lugar desde el inicio de la crisis.

Nuestro análisis también ha aportado aceptables respuestas a cuestiones como la volatilidad del ciclo que si bien se manifestaba con bastante claridad hasta 20052006, a partir de este último ejercicio ha registrado un cambio muy notable en el sentido de que se ha incrementado la volatilidad del ciclo español en la crisis. Por otra parte, el estudio de la coherencia y la sincronía cíclica ha permitido concluir 
que la productividad (laboral) española muestra una evolución bastante diferenciada antes y después de la crisis.

Uno de los aspectos más interesantes del trabajo es el referente al análisis sectorial de la productividad. Los resultados son, en síntesis, los siguientes: la agricultura se nos muestra como a-cíclica y retrasada hasta 2006 y pro-cíclica y adelantada a partir del inicio de la crisis. El sector manufacturero se muestra pro-cíclico antes y después de la crisis, si bien con una sincronía adelantada y retrasada en los dos subperíodos. Los servicios fueron $a$-cíclicos y su sincronía era coincidente en la fase expansiva, pero pasan a ser pro-cíclicos y adelantados entre 2007 y 2011. Por último, la construcción muestra un patrón de comportamiento prácticamente coincidente al de la economía en su conjunto, de forma que pasa de signo positivo a negativo en los dos sub-períodos considerados. Estos resultados ofrecen una conclusión interesante. El comportamiento atípico del ciclo de la productividad española está muy influenciado por el comportamiento de dos de las actividades en las que se ha basado el tejido productivo nacional en las últimas décadas: la construcción y algunas actividades de servicios destinadas al consumo final, ambas poco dinámicas y con fuertes procesos de destrucción de empleo en etapas recesivas como la actual.

Como hemos señalado al inicio de estas notas finales, son bastantes las cuestiones que deja abiertas este trabajo, que deben merecer nuevas investigaciones. Lo que nadie se atreverá a poner en duda es que la productividad es uno de los problemas clave que hay que abordar para que la economía española pueda crecer de forma sostenida en el futuro. La mejora que ha experimentado desde que se inició la crisis responde, básicamente, a la fuerte pérdida de empleos que ha tenido y sigue teniendo lugar y no a mejoras en términos de dotación de capital por trabajador, incorporación de nuevas tecnologías y cambios organizativos en las empresas, con incentivos para que la competitividad se convierta en el catalizador de un giro positivo y duradero de dicha variable. Como señalábamos al principio del artículo, la mejora sostenida de la productividad es clave para garantizar el crecimiento económico y el bienestar colectivo.

\section{Referencias bibliográficas}

[1] BBVA (2010). La productividad en España. Crecimiento y crisis. Cuadernos Fundación BBVA, Capital y Crecimiento, 3/2010, Madrid

[2] BBVA (2000): «Why productivity is procyclical. Why do we care?», Federal Reserve Bank of Chicago Working Paper, 2000-11, Federal Reserve Bank of Chicago, Chicago.

[3] BENCIVENGA, V. (1992): «An econometric study of hours and output variation with preference shocks», International Economic Review, 33 (2): 449-71.

[4] BENHABIB, J.; ROGERSON, R., y WRIGHT, R. (1991): «Homework in macroeconomics: Household production and aggregate fluctuations», Journal of Political Economy, 99 (1), 166-87.

[5] BLACKBURN, K., y RAVN, M. (1991): «Business cycles in the UK: facts and fictions», Economica, 59 (236): 383-401. 
[6] CAMAGNI, R. y CAPELLIN, R. (1985): La productivité sectorielle et la politique régionale, European Commission, Report. Bruselas.

[7] CHRISTODOULAKIS, N.; DIMELIS, S., y KOLLINTZAS, T. (1995): «Comparisons of business cycles in the EC: Idyosincracies and regularities», Economica, 62 (245), $1-27$.

[8] COGLEY, T. (1990): Spurious business cycle phenomena in HP detrended series. University of Washington, mimeo, Washington D.C.

[9] CUADRADO-ROURA, J. R., y ORTIZ, A. (2001): «Business cycle and service industries: General trends and the Spanish case», The Service Industries Journal, 21 (1), 103-122.

[10] CUADRADO-ROURA, J. R (dir.) y otros (2010): El sector construcción en España: análisis, perspectivas y propuestas. Ed. Colegio Libre de Eméritos, Madrid.

[11] DIMELIS, S. (2001): «Inventory investment over the business cycle in the EU and the US», International Journal of Production Economics, 71 (1): 1-8.

[12] FIORITO, R., y KOLLINTZAS, T. (1994): «Stylized facts of business cycles in the G-7 from a real business cycles perspective», European Economic Review, 38 (2), 235-69.

[13] FRENCH, M. (2005): «A nonlinear look at trend MFP growth and the business cycle: Results from a hybrid Kalman/Markov switching model», Finance and Economics Discussion Series, 2005-12, Federal Reserve Board, Washington D.C.

[14] GORDON, R. (2003): «Hi-tech innovation and productivity growth: Does supply create its own demand?», NBER Working Papers, 9437, NBER, Cambridge.

[15] HANSEN, G. (1985): «Indivisible labor and the business cycle», Journal of Monetary Economics, 16 (3): 309-29.

[16] HANSEN, G., y WRIGHT, R. (1992): «The labour market in real business cycle theory», Federal Reserve Bank of Minneapolis Quarterly Review, 16 (2): 2-12.

[17] HARVEY, A. C., y JAEGER, A. (1991): «De-trending stylised facts and the business cycle», Discussion Paper, EM/91/230, Suntory-Toyota International Center for Economics and Related Disciplines, Londres.

[18] HARVEY, A. C., y TRIMBUR, T. (2001): General model-based filters for extracting cycles and trends in economic time series. Cambridge University, mimeo, Cambridge.

[19] HODRICK, R., y PRESCOTT, E. C. (1997): «Post-war US business cycles: An empirical investigation», Journal of Money, Credit and Banking, 29 (1), 1-16.

[20] INKLAAR, R., y McGUCKIN, R. (2003): «Structural and cyclical performance», en M. O’Mahony y B. van Ark (eds.), EU Productivity and Competitiveness. An Industry Perspective (Chapter IV). Bruselas: European Commission.

[21] KALMAN, R. (1960): «A new approach to linear filtering and prediction problems», Journal of Basic Engineering, 82 (D): 35-45.

[22] KIM, S.; KOSE, M., y PLUMMER, M. (2003): «Dynamics of business cycle in Asia: Differences and similarities», Review of Development Economics, 7 (3), 462-77.

[23] KING, R., y REBELO, S. (1993): «Low frequency and real business cycles», Journal of Economic Dynamics and Control, 17 (1-2): 207-31.

[24] KYDLAND, F., y PRESCOTT, E.C. (1982): «Time to build and aggregate fluctuations», Econometrica, 50 (6): 1345-70.

[25] LUCAS, R. (1977): «Understanding business cycles», en K. Brunner y A. Meltzer (eds.), Stabilization of the Domestic and International Economy, Carnegie-Rochester Conference Series on Public Policy, 5, Amsterdam: North-Holland. 
[26] MAROTO,A. (2011): «Productivity growth and cyclical dynamics in the service sector. The Spanish case», Service Industries Journal, 31 (5), 725-745.

[27] MAROTO,A., y CUADRADO-ROURA, J. R. (2006): La productividad en la economía española, Colección Estudios, Instituto de Estudios Económicos, Madrid.

[28] MAROTO, A., y CUADRADO-ROURA, J. R. (2009): «Is tertiarization an obstacle to productivity growth? A comparative analysis», Structural Change and Economic Dynamics, 20 (4), 254-265.

[29] MAROTO, A., y RUBALCABA, L. (2008): «Services productivity revisited», Service Industries Journal, 28 (3), 337-53.

[30] McCALLUM, B. (1989): «Real business cycle analysis», en R. Barro (ed.), Modern business cycle theory (Chapter 1, pp. 63-81). Cambridge, MA: Harvard University Press.

[31] O’MAHONY, M., y VAN ARK, B. (2003): EU productivity and competitiveness: An industry perspective. Can Europe resume the catching-up process? Bruselas: European Commission Enterprise publications.

[32] PRESCOTT, E. C. (1986): Theory ahead business cycle measurement. Federal Reserve Bank of Minneapolis Quarterly Review, 10 (4): 9-22.

[33] RAVN, M., y UHLIG, H. (2002): «On adjusting the HP Filter for the frequency of observations», Review of Economics and Statistics, 84 (2), 371-80.

[34] ROSENTHAL, R. (1991): Meta-analytic procedures for social research. Newbury Park, CA: Sage Publications Inc.

[35] WELCH, G., y BISHOP, G. (1995): «An introduction to Kalman filter», Technical Report, TR95-041, University of North Carolina, Chapel Hill, NC.

[36] ZARNOWITZ, V., y OZYILDIRIM, A. (2001): «Time series decomposition and measurement of business cycles, trends and growth cycles», Conference Board Working Paper, 01-04, The Conference Board, Washington D.C. 